UMN-TH-2227/03

December 2003

\title{
Dirac Neutrino Masses with Planck Scale Lepton Number Violation
}

\author{
Tony Gherghetta \\ School of Physics and Astronomy \\ University of Minnesota \\ Minneapolis, MN 55455, USA
}

\begin{abstract}
It is shown how pure Dirac neutrino masses can naturally occur at low energies even in the presence of Planck scale lepton number violation. The geometrical picture in five dimensions assumes that the lepton number symmetry is explicitly broken on the Planck brane while the right-handed neutrino is localised on the $\mathrm{TeV}$ brane. This physical separation in the bulk causes the global lepton number to be preserved at low energies. A small wavefunction overlap between the left-handed and right-handed neutrinos then naturally leads to a small Dirac Yukawa coupling. By the AdS/CFT correspondence there exists a purely four-dimensional dual description in which the right-handed neutrino is a composite CFT bound state. The global lepton number is violated at the Planck scale in a fundamental sector whose mixing into the composite sector is highly suppressed by CFT operators with large anomalous dimensions. A similar small mixing is then also responsible for generating a naturally small Dirac Yukawa coupling between the fundamental left-handed neutrino and the composite righthanded neutrino.
\end{abstract}




\section{Introduction}

Recent neutrino oscillation experiments have spectacularly confirmed that neutrinos have a small nonzero mass. However it remains an open question as to whether the neutrino mass is of the Majorana or Dirac type. Historically the preference is that they are of the Majorana type, because a small mass can be elegantly explained by the see-saw mechanism [1]. But this comes at the expense of introducing a new mass scale below the Planck scale, which presumably is related to the usual GUT scale. Since there is no experimental evidence for this picture it behooves us to consider the possibility that neutrinos could be Dirac particles.

The usual argument against Dirac neutrino masses consists of requiring unnaturally small Yukawa couplings, although this argument seems presumptuous especially given the fact that we do not understand why the electron Yukawa coupling is so small. Furthermore, one must impose a global lepton number symmetry to forbid a Majorana mass term for the right-handed neutrino. However this global symmetry can be badly broken by black hole physics, and consequently for any consistent theory of quantum gravity. In fact, in string theory it is not possible to obtain an additive global conservation law 2]. These symmetry violating effects at an ultraviolet (UV) scale $M$ can be parametrised by including all lepton number violating operators in the Lagrangian. If we introduce the righthanded Weyl neutrino $\nu_{R}$, which is a standard model singlet carrying one unit of lepton number, then the dimension three Majorana mass term

$$
M\left(\nu_{R} \nu_{R}+\text { c.c. }\right)
$$

is the most relevant lepton number violating operator. At low energies we can integrate out this right-handed neutrino state. This then leads to the usual seesaw mechanism and to the Majorana nature of the physical neutrino mass. Thus, in the presence of Planck scale lepton number violating effects it would seem to be difficult to maintain the Dirac nature of the neutrino.

In this paper we show that the above incompatibility can be naturally avoided in a warped extra dimension, or strongly coupled conformal field theory (CFT). We will argue that if the UV physics violates the lepton number symmetry maximally, then this symmetry will still be restored in the infrared (IR), where the physical neutrino is a Dirac particle. So the usual argument that the Standard Model has an accidental global lepton number symmetry at low energies because all lepton number violating operators are irrelevant will be extended to the case

of the Standard Model plus a right-handed neutrino. While it will be easy to 
visualise our scenario in a warped extra dimension, remarkably we will see that because of the AdS/CFT correspondence [3] the geometrical mechanism of the extra dimension can be completely interpreted in four dimensions where the righthanded neutrino is a CFT bound state ${ }^{1}$. Since the CFT is a large $N$ strongly coupled gauge theory, the coupling of the composite state to the UV violation of lepton number involves a form factor $F\left(q^{2}\right)$ which vanishes in the limit $q^{2} \rightarrow \infty$. In fact the operator responsible for creating a right-handed neutrino acquires a large anomalous dimension. This causes the usual dimension three operator (11) to be highly suppressed in the IR and the right-handed neutrino does not decouple. In other words lepton number violation is only characterised by irrelevant operators, leading to a theory containing a right-handed neutrino with an accidental global lepton number symmetry in the IR. A similar mechanism occurs for global supersymmetry, which can be maximally broken on the Planck brane, while fields confined to the $\mathrm{TeV}$ brane (such as the Higgs scalar field) remain supersymmetric [5]. Other interesting possibilities have also been discussed in Refs [6, 7, 8, 9].

Neutrino masses in extra dimensions were first considered in flat space in Ref. [10]. The generalisation to warped extra dimensions was studied by Grossman and Neubert [11, where a Majorana mass for the right-handed neutrino was forbidden by imposing a global lepton number symmetry. But since the right-handed neutrino is localised on the Planck brane one would expect that the global symmetry breaking effects at the Planck scale will lead to Majorana neutrino masses, and not Dirac masses. More recently in Ref. 12, 13, a Majorana mass for the right-handed neutrino was introduced on the Planck brane in order to obtain Majorana neutrino masses of the right magnitude via the usual seesaw mechanism. Instead by localising the right-handed neutrino towards the $\mathrm{TeV}$ brane we will see that Dirac neutrino masses can be naturally generated even in the presence of a large lepton number violating term on the Planck brane. The effective right-handed Majorana mass can be naturally made to vanish.

Let us first begin with the geometrical picture of our setup in five dimensions. This will make it easy to visualise how the Dirac nature of the neutrino can be naturally obtained. Consider a slice of $\mathrm{AdS}_{5}$ where the fifth dimension is compactified on an orbifold $S^{1} / Z_{2}$ of radius $R$ with $0 \leq y \leq \pi R$. The metric solution is given by

$$
d s^{2}=e^{-2 k|y|} \eta_{\mu \nu} d x^{\mu} d x^{\nu}+d y^{2},
$$

where $k$ is the AdS curvature scale, and the Minkowski metric $\eta_{\mu \nu}$ has signature

\footnotetext{
${ }^{1}$ In purely four dimensions composite right-handed neutrinos generating naturally small Dirac masses were also considered in Ref. 4].
} 
$(-+++)$. At the orbifold fixed points $y^{*}=0$ and $y^{*}=\pi R$ there are two 3branes, the Planck brane and $\mathrm{TeV}$ brane, respectively. For each neutrino Weyl fermion, $\nu_{L, R}$ we will associate a five dimensional (5D) Dirac fermion field $\Psi_{L, R}$ with components

$$
\Psi_{i}\left(x^{\mu}, y\right)=\left(\begin{array}{c}
\psi_{1 i}\left(x^{\mu}, y\right) \\
\bar{\psi}_{2 i}\left(x^{\mu}, y\right)
\end{array}\right)
$$

where $i=L, R$. We will assume a separation of variables for the wavefunctions $\psi_{k i}(x, y)=1 / \sqrt{\pi R} \sum_{n} \psi_{k i}^{(n)}(x) f_{k i}^{(n)}(y)$ so that upon compactification on the orbifold $S^{1} / Z_{2}$ the zero modes, $\psi_{1 i}^{(0)}$ will become the neutrino states $\nu_{L, R}$, while the zero modes, $\psi_{2 i}^{(0)}$ are projected out. At the massive Kaluza-Klein level each fermion $\psi_{1 i}^{(n)}$ pairs up with the fermion $\psi_{2 i}^{(n)}$ to form massive vector-like Dirac states. Assume that the action for the bulk fermion fields $\Psi_{i}$ in the warped space is given by

$$
S=-\int d^{4} x \int d y \sqrt{-g}\left[i \bar{\Psi}_{i} \Gamma^{M} D_{M} \Psi_{i}+i c_{i} k \epsilon(y) \bar{\Psi}_{i} \Psi_{i}+i \frac{b_{M}}{2} \delta(y)\left(\bar{\Psi}_{R}^{c} \Psi_{R}+\text { h.c. }\right)\right],
$$

where $c_{i}, b_{M}$ are dimensionless constants which parametrise the bulk Dirac and boundary Majorana mass terms, respectively. The kinetic term in (4) contains the gamma matrices $\Gamma^{M}$ defined in curved space, and the covariant derivative $D_{M}=\partial_{M}+\omega_{M}$, where $\omega_{M}$ is the spin connection (see Ref [14]). When $b_{M}=0$ the canonically normalised zero mode wavefunction is determined to be [14]

$$
f_{1 i}^{(0)}(y)=\frac{1}{N_{0}} e^{\left(2-c_{i}\right) k|y|},
$$

where $N_{0}$ is a normalisation constant. The specific value of $c_{i}$ parametrising the bulk Dirac mass term determines the degree of localisation of the zero mode field in the extra dimension. When $c_{i}>1 / 2\left(c_{i}<1 / 2\right)$ the zero mode is localised towards the Planck (TeV) brane. The bulk Dirac mass term provides a convenient way to localise the zero mode fields at any position in the extra dimension.

In this $5 \mathrm{D}$ geometrical framework suppose that the left-handed neutrino $\nu_{L}$ is localised towards the Planck brane and parametrised by the bulk mass parameter $c_{L}$, while the right-handed neutrino $\nu_{R}$ is localised towards the $\mathrm{TeV}$ brane, and parametrised by the bulk mass parameter $c_{R}$. On the Planck brane the source of lepton number violation will be parametrised by a Majorana mass term with nonzero $b_{M}$ in (4) for the right-handed neutrino. In the presence of this lepton number violation the right-handed neutrino zero mode field will no longer remain massless. However, since the zero mode is assumed to be localised towards the $\mathrm{TeV}$ brane we expect the zero mode will only acquire a small mass depending on how well the field is localised. 
To calculate the zero mode mass in the presence of the Majorana boundary term we will consider the classical equations of motion for the right-handed neutrino Dirac spinor $\Psi_{R}$. These are given by

$$
\begin{aligned}
& i e^{k|y|} \bar{\sigma}^{\mu} \partial_{\mu} \widehat{\nu}_{2 R}+\left(\partial_{5}+c_{R} k \epsilon(y)\right) \widehat{\widehat{\nu}}_{1 R}=0, \\
& i e^{k|y|} \bar{\sigma}^{\mu} \partial_{\mu} \widehat{\nu}_{1 R}-\left(\partial_{5}-c_{R} k \epsilon(y)\right) \widehat{\bar{\nu}}_{2 R}+b_{M} \delta(y) \widehat{\bar{\nu}}_{1 R}=0,
\end{aligned}
$$

where we have absorbed the spin connection term by defining $\widehat{\nu}_{i R}=e^{-2 k|y|} \nu_{i R}$. Assuming that the solutions have the form $\widehat{\nu}_{i R}(x, y)=1 / \sqrt{\pi R} \sum \nu_{i R}^{(n)}(x) \hat{f}_{i R}^{(n)}(y)$ we obtain [14]

$$
\begin{aligned}
& f_{1 R}^{(n)}(y)=\frac{e^{\frac{5}{2} k|y|}}{N_{1 n}}\left[J_{\alpha_{1}}\left(\frac{m_{n}}{k e^{-k|y|}}\right)-\frac{J_{\alpha_{1}+1}\left(\frac{m_{n}}{k e^{-\pi k R}}\right)}{Y_{\alpha_{1}+1}\left(\frac{m_{n}}{k e^{-\pi k R}}\right)} Y_{\alpha_{1}}\left(\frac{m_{n}}{k e^{-k|y|}}\right)\right], \\
& f_{2 R}^{(n)}(y)=\epsilon(y) \frac{e^{\frac{5}{2} k|y|}}{N_{2 n}}\left[J_{\alpha_{2}}\left(\frac{m_{n}}{k e^{-k|y|}}\right)-\frac{J_{\alpha_{2}}\left(\frac{m_{n}}{k e^{-\pi k R}}\right)}{Y_{\alpha_{2}}\left(\frac{m_{n}}{k e^{-\pi k R}}\right)} Y_{\alpha_{2}}\left(\frac{m_{n}}{k e^{-k|y|}}\right)\right],
\end{aligned}
$$

where $\alpha_{1,2}=\left|c_{R} \pm \frac{1}{2}\right|, N_{k n}$ are normalisation constants, and at $y=\pi R$ we have imposed even (odd) boundary conditions on the fermions, $f_{1 R}\left(f_{2 R}\right)$. The Kaluza-Klein mass spectrum $m_{n}$ is obtained by taking into account the boundary Majorana mass at $y=0$, and leads to the boundary condition

$$
f_{2 R}^{(n)}(0)=\frac{b_{M}}{2} f_{1 R}^{(n)}(0)
$$

This gives rise to the lightest mass mode

$$
m_{0} \simeq \frac{b_{M}}{2}\left(1-2 c_{R}\right) k e^{-\left(1-2 c_{R}\right) \pi k R}
$$

where we are assuming $c_{R}<-1 / 2$. This formula is analogous to that obtained for a boundary gaugino mass [15, [5], and appears in Refs [13, 16] which also considered a boundary Majorana neutrino mass. The masses of the Kaluza-Klein modes $\nu_{1 R}^{(n)}$ and $\nu_{2 R}^{(n)}$ which normally form Dirac pairs, can also be obtained by solving (10). They are separated by a mass gap of order the TeV scale, and obtain an exponentially suppressed Majorana mass contribution from the boundary mass at $y=0$.

Thus, we see that in the presence of a boundary Majorana mass the zero mode obtains a Majorana mass (11). However, even though the lepton number violation is of order the Planck scale, the Majorana mass (111) for the right handed neutrino is exceedingly small assuming that $k e^{-\pi k R}=\mathrm{TeV}$. In fact if the righthanded neutrino is completely localised on the $\mathrm{TeV}$ brane, corresponding to the 
formal limit of $c_{R} \rightarrow-\infty$, then the right-handed neutrino remains massless. This is because there are no direct couplings to the Planck brane, and any source of lepton number violation in the UV. Note that if instead the Majorana mass parameter on the Planck brane $b_{M} \rightarrow \infty$, then the lowest lying states are two degenerate singlet Weyl neutrinos forming a Dirac particle state with mass

$$
m_{0} \simeq \pm \sqrt{4 c_{R}^{2}-1} k e^{-\left(\frac{1}{2}-c_{R}\right) \pi k R}
$$

where $c_{R}<-1 / 2$. Even though this mass can be made arbitrarily small, it includes an extra Weyl neutrino $\nu_{2}$ (besides $\nu_{1 R}$ ).

We have seen that the right-handed Weyl neutrino $\nu_{R}$ remains massless in the presence of lepton number violation on the UV brane. However we still need to generate a neutrino mass. This is accomplished by writing a bulk Yukawa interaction between the left-handed neutrino $\nu_{L}$, right-handed neutrino $\nu_{R}$, and the Higgs field, $H$. This generates a Dirac neutrino mass where we will assume that the right-handed neutrino is sufficiently localised on the $\mathrm{TeV}$ brane, so that its lepton number violating Majorana mass is negligibly small.

For simplicity consider the original Randall-Sundrum model [17, where a completely localised Higgs field breaks electroweak symmetry on the TeV brane. This generates a Dirac mass term on the TeV brane from the Yukawa interaction

$$
\int d^{4} x \int d y \lambda_{5} \nu_{L}(x, y) \nu_{R}(x, y) H(x) \delta(y-\pi R)
$$

where $\lambda_{5}$ is the 5D Yukawa coupling. When the Higgs field obtains a vacuum expectation value, $\langle H\rangle=v$, the effective four dimensional Yukawa coupling is given by [14]

$$
\lambda_{4}=\frac{\lambda_{5} k}{N_{L} N_{R}} e^{\left(1-c_{L}-c_{R}\right) \pi k R} \simeq \lambda_{5} k \sqrt{\left(c_{L}-1 / 2\right)\left(1 / 2-c_{R}\right)} e^{\left(\frac{1}{2}-c_{L}\right) \pi k R},
$$

where $1 / N_{i}^{2}=\left(1 / 2-c_{i}\right) /\left(e^{\left(1-2 c_{i}\right) \pi k R}-1\right)$, and $c_{L}>1 / 2, c_{R}<-1 / 2$. Assuming that $\lambda_{5} k \sim 1$, we see that the Dirac neutrino mass

$$
m_{\nu}=\lambda_{4} v \sim 10^{-2} \mathrm{eV}
$$

is naturally obtained for $c_{L} \sim 1.36$, where we have taken $c_{R}<-1 / 2$ and $\pi k R=$ 34.54. Thus, in the IR where lepton number is a good symmetry the neutrino is a Dirac particle and the magnitude of its mass can naturally be obtained via a small wavefunction overlap. Since the right-handed neutrino lives on the $\mathrm{TeV}$ brane it is not sensitive to any UV violation of global lepton number symmetry. 
Note also that the Yukawa coupling (14) does not exponentially depend on the right-handed bulk Dirac mass parameter $c_{R}$. This is because the right-handed field is assumed to be localised towards the $\mathrm{TeV}$ brane, so that the wave function overlap with the localised Higgs field is of order one. This of course means that in this simple setup the Yukawa coupling will generically be the same for each fermion in the left-handed doublet unless there is a hierarchy in the bulk mass parameters $c_{\nu_{R}} \sim 10^{-14} c_{e_{R}}$. However, a set up with no hierarchies can easily be obtained by delocalising the Higgs field in a supersymmetric framework [14, 18. For example, requiring $c_{H}=0.5, c_{L}=1.32, c_{e_{R}} \simeq 0.2$, and $c_{\nu_{R}} \simeq-2$, leads to an electron Yukawa coupling, $\lambda_{e} \sim 10^{-6}$ and an electron-neutrino Yukawa coupling, $\lambda_{\nu_{e}} \sim 10^{-13}$. Alternatively, for $c_{H}=0.11, c_{L}=1.32$ the Yukawa couplings are obtained for $c_{e_{R}} \simeq 1$ and $c_{\nu_{R}} \simeq-2.5$. A solution can always be found with $c_{\nu_{R}}$ large and negative, so that the right handed neutrino is localised towards the $\mathrm{TeV}$ brane, away from the UV violation of lepton number on the Planck brane.

\section{AdS/CFT correspondence}

Remarkably the 5D geometric picture can be given a purely 4D description via the AdS/CFT correspondence [3]. This correspondence relates the 5D AdS theory to a large $N$ strongly coupled $4 \mathrm{D}$ CFT. The UV brane at $y=0$ corresponds to a UV cutoff at momentum $p=k$ in the $4 \mathrm{D}$ CFT, while the boundary at $y=\pi R$ corresponds to an IR cutoff at $p=k e^{-\pi k R}$ [19, 20]. In this way we see that the slice of $\mathrm{AdS}_{5}$ provides a dual description to a slice in momentum space of the large $N$ strongly coupled CFT. The conformal symmetry is spontaneously broken in the IR leading to the formation of CFT bound states, much like the mesons and baryons in QCD. In fact, fields which are localised towards the TeV brane are interpreted as bound states of the CFT, while fields which are localised towards the Planck brane are understood to be fundamental states which must be added to the CFT. In particular the Kaluza-Klein spectrum which is always localised towards the IR brane, corresponds to the infinite number of bound states of the strongly coupled CFT which are weakly coupled for large $N$ [21]. In general the $y=0$ boundary value of $5 \mathrm{D}$ bulk fields $\Phi$ are identified as sources of CFT operators $\mathcal{O}$ via the term

$$
\mathcal{L}=\lambda \Phi(x) \mathcal{O}(x)
$$

where the mass of the bulk field is related to the dimension of the CFT operator.

The case of global lepton number symmetry is very similar to what occurs with global supersymmetry [5]. In particular let us consider the case of a bulk fermion 
$\Psi$. Only half the degrees of freedom of the bulk Dirac fermion are identified as sources on the AdS boundary 22]. The four-dimensional theory consists of the CFT sector fields and the fundamental sector source fields. The physical mass eigenstates in the 4D dual theory are a linear superposition of the CFT sector fields and the fundamental source fields. The global lepton number symmetry is only a symmetry of the CFT sector, whereas the fundamental sector explicitly breaks the global symmetry.

Consider first the bulk Dirac fermion field $\Psi_{L}$ containing the left-handed neutrino field. In the $4 \mathrm{D}$ dual theory we introduce the fundamental source field $\psi_{L}$ and for $c_{L}>1 / 2$ we have

$$
\mathcal{L}=\mathcal{L}_{C F T}+i \bar{\psi}_{L} \bar{\sigma} \cdot \partial \psi_{L}+\xi k^{1 / 2-c_{L}} \bar{\psi}_{L} \mathcal{O}_{L}+\text { h.c. }+\ldots
$$

where $\xi$ is a dimensionless coupling and $\mathcal{O}_{L}$ is a composite fermion operator which couples to $\psi_{L}$ with $\operatorname{dim} \mathcal{O}_{L}=3 / 2+\left|c_{L}+1 / 2\right|$ [7]. When $c_{L}>1 / 2$ the coupling which mixes the fundamental sector with the CFT sector is always irrelevant. From the 5D theory we know that the physical neutrino $\nu_{L}$ is localised towards the Planck brane, so that in the dual theory this is interpreted by saying that the physical mass eigenstate is predominantly composed of the fundamental sector state, $\psi_{L}$.

Similarly for the bulk Dirac fermion field $\Psi_{R}$ containing the right-handed neutrino field. In this case the dual theory Lagrangian for $c_{R}<-1 / 2$ is given by

$$
\mathcal{L}=\mathcal{L}_{C F T}+\xi k^{1 / 2+c_{R}} \bar{\psi}_{R} \mathcal{O}_{R}+\text { h.c. }+\ldots,
$$

where $\psi_{R}$ is a fundamental source field which couples to the CFT operator $\mathcal{O}_{R}$ with $\operatorname{dim} \mathcal{O}_{R}=3 / 2+\left|c_{R}-1 / 2\right|$ [7]. The physical right-handed neutrino is localised towards the $\mathrm{TeV}$ brane, and we see that in the $4 \mathrm{D}$ dual theory the mixing with the fundamental source field $\psi_{R}$ is highly suppressed in the limit $c_{R} \rightarrow-\infty$. This means that the physical mass eigenstate $\nu_{R}$ does not couple to the fundamental sector. Below the TeV scale the right-handed neutrino $\nu_{R}$ is a composite CFT bound state.

Normally in four dimensions the dimension three operator (11) will decouple the right-handed neutrino at low energies, and the light neutrinos are necessarily Majorana. Let us now understand why this is no longer the case in the $4 \mathrm{D}$ dual CFT theory. In the 5D theory lepton number symmetry was only broken on the Planck brane, and using the AdS/CFT dictionary, this corresponds to breaking lepton number symmetry at the Planck scale in the 4D dual description. Only the fundamental sector can feel this explicit UV breaking, while the global lepton number symmetry is preserved in the CFT sector. This means that the composite 
right-handed neutrino will only feel the explicit breaking via the mixing term in (18), where for the fundamental state $\psi_{R}$ we include a mass term

$$
\frac{k}{2 b_{M}} \psi_{R} \psi_{R}+\text { h.c. }
$$

In the CFT the operator $\mathcal{O}_{R}$ has the appropriate quantum numbers to create the right-handed neutrino composite states, $\psi_{\mathcal{O}_{R}}^{(n)}$. We will assume that composite particle states will appear at the scale $\mu$, where the conformal symmetry is spontaneously broken. Thus by writing $\mathcal{O}_{R} \simeq \mu^{1 / 2-c_{R}} \psi_{\mathcal{O}_{R}}^{(0)}$ at $q^{2} \simeq 0$ for the massless resonance, the mixing matrix with the fundamental field $\psi_{R}$ is given by

$$
\begin{aligned}
& \left(\bar{\psi}_{R}, \psi_{\mathcal{O}_{R}}^{(0)}\right)\left(\begin{array}{cc}
\frac{k}{2 b_{M}} & \frac{\xi}{2} k\left(\frac{\mu}{k}\right)^{\frac{1}{2}-c_{R}} \\
\frac{\xi}{2} k\left(\frac{\mu}{k}\right)^{\frac{1}{2}-c_{R}} & 0
\end{array}\right)\left(\begin{array}{c}
\bar{\psi}_{R} \\
\psi_{\mathcal{O}_{R}}^{(0)}
\end{array}\right)+\text { h.c. } \\
& \simeq\left(\bar{\psi}_{R}^{\prime}, \psi_{\mathcal{O}_{R}}^{(0) \prime}\right)\left(\begin{array}{cc}
\frac{k}{2 b_{M}}+\ldots & 0 \\
0 & -\frac{\xi^{2}}{2} b_{M} k\left(\frac{\mu}{k}\right)^{1-2 c_{R}}+\ldots
\end{array}\right)\left(\begin{array}{c}
\bar{\psi}_{R}^{\prime} \\
\psi_{\mathcal{O}_{R}}^{(0) \prime}
\end{array}\right)+\text { h.c. }
\end{aligned}
$$

where we have assumed that $\mu \ll k$ and in terms of the original fields we obtain

$$
\begin{gathered}
\bar{\psi}_{R}^{\prime} \simeq \bar{\psi}_{R}+b_{M} \xi\left(\frac{\mu}{k}\right)^{\frac{1}{2}-c_{R}} \psi_{\mathcal{O}_{R}}^{(0)}, \\
\psi_{\mathcal{O}_{R}}^{(0) \prime} \simeq \psi_{\mathcal{O}_{R}}^{(0)}-b_{M} \xi\left(\frac{\mu}{k}\right)^{\frac{1}{2}-c_{R}} \bar{\psi}_{R} .
\end{gathered}
$$

Thus, the dimension three Majorana mass operator at the scale $\mu$ becomes for $c_{R}<-1 / 2$

$$
\mathcal{L}_{\text {Majorana }} \simeq b_{M}\left(\frac{\mu}{k}\right)^{1-2 c_{R}} k \nu_{R} \nu_{R}+\text { h.c. }
$$

where $\nu_{R}$ is associated with the composite field $\psi_{\left.\mathcal{O}_{R}\right)}^{(0)}$. Assuming that $\mu=k e^{-\pi k R}$ then we obtain the result consistent with (11). In the limit that $c_{R} \rightarrow-\infty$ we see that the physical right-handed neutrino is mostly a composite state, and the Majorana mass is highly suppressed so that the dimension three operator becomes irrelevant in the IR. Consequently, at low energies global lepton number symmetry is restored.

In the case where the boundary Majorana mass parameter $b_{M} \rightarrow \infty$, the zero mode does not couple to the Planck brane which means that the fundamental source field is massless. The mixing matrix becomes

$$
\left(\bar{\psi}_{R}, \psi_{\mathcal{O}_{R}}^{(0)}\right)\left(\begin{array}{cc}
0 & \frac{\xi}{2} k\left(\frac{\mu}{k}\right)^{\frac{1}{2}-c_{R}} \\
\frac{\xi}{2} k\left(\frac{\mu}{k}\right)^{\frac{1}{2}-c_{R}} & 0
\end{array}\right)\left(\begin{array}{c}
\bar{\psi}_{R} \\
\psi_{\mathcal{O}_{R}}^{(0)}
\end{array}\right)+h . c .
$$


and give rises to masses $\pm \frac{\xi}{2} k e^{-\left(\frac{1}{2}-c_{R}\right) \pi k R}$, which form a Dirac mass that is consistent with the bulk calculation (12).

Finally, using the AdS/CFT dictionary it is straightforward to understand the smallness of the Dirac neutrino Yukawa coupling in the 4D dual theory. The Higgs is assumed to be localised on the TeV brane and corresponds to a CFT bound state much like the right-handed neutrino. In a large $N$ gauge theory the trilinear meson vertex $\Gamma_{3}$, corresponding to a Yukawa interaction between three composite states is $\Gamma_{3} \sim 1 / \sqrt{N}$ [21. However, the left-handed neutrino state is localised towards the Planck brane, which means that the physical left-handed neutrino is primarily composed of the fundamental state. Thus, in the Yukawa interaction we need to take into account the mixing term (17) and the matrix element $\left\langle 0\left|\mathcal{O}_{L}\right| \psi_{\mathcal{O}_{L}}^{(n)}\right\rangle \sim \sqrt{N}$. At the scale $\mu$ this leads to the Yukawa interaction

$$
\mathcal{L}_{\text {Yukawa }} \simeq\left(\frac{\mu}{k}\right)^{c_{L}-\frac{1}{2}} \nu_{L} \nu_{R} H+\text { h.c. }
$$

Assuming that the conformal symmetry is broken at the scale $\mu=k e^{-\pi k R}$ then we obtain the four-dimensional Yukawa coupling consistent with the bulk calculation (14). In the dual CFT picture we see that the Yukawa coupling is naturally suppressed because the mixing between the fundamental sector and CFT sector is very small. It is also easy to see that the suppression only depends on $c_{L}$ or the localisation of the left-handed neutrino. The CFT operators $\mathcal{O}_{i}$ create the composite bound states and since both the Higgs and right-handed neutrino are primarily CFT bound states the mixing contribution from the fundamental sector is extremely suppressed for these fields. Again this is similar to what happens for global supersymmetry [5].

\section{Conclusion}

If the standard model is augmented with a right-handed singlet neutrino $\left(\nu_{R}\right)$ then we have shown that the neutrino masses can be purely Dirac even in the presence of an explicit UV breaking of lepton number. In five dimensions this can be understood by requiring that lepton number is explicitly broken on the UV brane while the right-handed neutrino is localised on the IR brane. The fact that the right-handed neutrino is physically separated in the bulk from the source of lepton number violation means that the lepton number symmetry is preserved at low energies. Remarkably there exists a purely four-dimensional description of this model. At the $\mathrm{TeV}$ scale the right-handed neutrino is predominantly a 
CFT bound state which contains a tiny admixture of a fundamental state. In the CFT sector the lepton number symmetry is preserved while the symmetry is explicitly broken in the fundamental sector. The extremely tiny mixing with the fundamental sector accounts for the preservation of lepton number symmetry at low energies. In this setup there is no need to introduce an intermediate mass scale since the neutrino masses are purely Dirac, and the smallness of the Yukawa couplings is explained by a small wavefunction overlap or large anomalous dimensions of CFT operators. In addition our model differs from previous Dirac neutrino models in extra dimensions in that we do not assume that the fundamental theory in the UV preserve a global lepton number symmetry. This removes the theoretical impediment of requiring that a consistent theory of quantum gravity preserve a global quantum number, and provides an alternative to the usual seesaw mechanism of Majorana neutrino masses.

\section{Acknowledgments}

I would like to thank K.S. Babu, A. Pomarol, M. Shifman, A. Vainshtein and M. Voloshin for useful discussions. This research was supported in part by a DOE grant DE-FG02-94ER40823 at the University of Minnesota, and by a grant from the Office of the Dean of the Graduate School of the University of Minnesota.

\section{References}

[1] M. Gell-Mann, P. Ramond and R. Slansky, in Supergravity, eds P. van Niewenhuizen and D.Z. Freedman (North Holland, Amsterdam 1980); T. Yanagida, in Proc. of Workshop on Unified Theory and Baryon Number in the Universe, eds. O. Sawada and A. Sugamoto, KEK, Tsukuba, (1979); S.L. Glashow, in Quarks and Leptons, Cargése lectures, ed. M. Lévy, (Plenum, 1980, New York); R. N. Mohapatra and G. Senjanovic, Phys. Rev. Lett. 44, 912 (1980).

[2] E. Witten, Nucl. Phys. Proc. Suppl. 91 (2000) 3 arXiv:hep-ph/0006332.

[3] J. M. Maldacena, Adv. Theor. Math. Phys. 2, 231 (1998) arXiv:hep-th/9711200; E. Witten, Adv. Theor. Math. Phys. 2, 253 (1998) arXiv:hep-th/9802150; S. S. Gubser, I. R. Klebanov and A. M. Polyakov, Phys. Lett. B 428, 105 (1998) arXiv:hep-th/9802109. 
[4] N. Arkani-Hamed and Y. Grossman, Phys. Lett. B 459, 179 (1999) arXiv:hep-ph/9806223.

[5] T. Gherghetta and A. Pomarol, Phys. Rev. D 67, 085018 (2003) arXiv:hep-ph/0302001.

[6] M. A. Luty, Phys. Rev. Lett. 89, 141801 (2002) arXiv:hep-th/0205077.

[7] R. Contino, Y. Nomura and A. Pomarol, arXiv:hep-ph/0306259.

[8] H. S. Goh, M. A. Luty and S. P. Ng, arXiv:hep-th/0309103.

[9] M. J. Strassler, arXiv:hep-th/0309122,

[10] K. R. Dienes, E. Dudas and T. Gherghetta, Nucl. Phys. B 557, 25 (1999) arXiv:hep-ph/9811428; N. Arkani-Hamed, S. Dimopoulos, G. R. Dvali and J. March-Russell, Phys. Rev. D 65, 024032 (2002) arXiv:hep-ph/9811448.

[11] Y. Grossman and M. Neubert, Phys. Lett. B 474, 361 (2000) arXiv:hep-ph/9912408.

[12] W. D. Goldberger, Y. Nomura and D. R. Smith, Phys. Rev. D 67, 075021 (2003) arXiv:hep-ph/0209158; Y. Nomura, JHEP 0311, 050 (2003) arXiv:hep-ph/0309189.

[13] S. J. Huber and Q. Shafi, arXiv:hep-ph/0309252.

[14] T. Gherghetta and A. Pomarol, Nucl. Phys. B 586, 141 (2000) arXiv:hep-ph/0003129.

[15] D. Marti and A. Pomarol, Phys. Rev. D 64, 105025 (2001) arXiv:hep-th/0106256.

[16] C. Csaki, C. Grojean, J. Hubisz, Y. Shirman and J. Terning, arXiv:hep-ph/0310355.

[17] L. Randall and R. Sundrum, Phys. Rev. Lett. 83, 3370 (1999) arXiv:hep-ph/9905221.

[18] T. Gherghetta and A. Pomarol, Nucl. Phys. B 602, 3 (2001) arXiv:hep-ph/0012378.

[19] N. Arkani-Hamed, M. Porrati and L. Randall, JHEP 0108, 017 (2001) arXiv:hep-th/0012148. 
[20] R. Rattazzi and A. Zaffaroni, JHEP 0104, 021 (2001) arXiv:hep-th/0012248; ; M. Perez-Victoria, JHEP 0105, 064 (2001) arXiv:hep-th/0105048.

[21] E. Witten, Nucl. Phys. B 160, 57 (1979).

[22] M. Henningson and K. Sfetsos, Phys. Lett. B 431, 63 (1998) arXiv:hep-th/9803251; W. Muck and K. S. Viswanathan, Phys. Rev. D 58, 106006 (1998) arXiv:hep-th/9805145|; J. Garriga and A. Pomarol, Phys. Lett. B 560, 91 (2003) |arXiv:hep-th/0212227. 